\title{
Meßaufbau zur Varaktoruntersuchung im R-Band
}

\author{
Test Set for Varactor Characterization at R-Band
}

\author{
Von Rainer Geißler
}

\author{
Mitteilung aus dem Institut für Hochfrequenztechnik der UNIVERSITÄT HANNOVER
}

Übersicht:

Mit Hilfe von Transmissions- und Reflexionsmessungen können die Elemente der Ersatzschaltung einer in einem Gehäuse eingebauten Varaktordiode gefunden werden. Durch Messung des Richtstromes und der Sperrspannung der Diode ist es auch möglich, die $C(U)$-Kennlinie, die dynamische Güte (bei Stromsteuerung) und den Sperrschichtstrom der Diode zu bestimmen. Die Konstruktion der dafür notwendigen Mikrowellenkomponenten (kontinuierlicher Hohlleiterübergang, Diodenhalter, koaxiales Sperrfilter) wird beschrieben.

\section{Abstract:}

The elements in the equivalent circuit of a packaged varactor can be determined by transmission and reflection measurements. By measuring the diode current and the back bias it's also possible to determine the $C(U)$-characteristic, the dynamic quality (for current control) and the junction current of the diode. The construction of the required microwave components (waveguide taper, diode mounting support, choke) is described.

Für die Dokumentation:

Varaktormessung / Stromsteuerung / R-Band-Komponente

\section{Einleitung}

Parametrische Mischer- und Verstärkeranordnungen im mm-Wellengebiet werden sinnvollerweise mit einem Ersatzschaltbild für Stromsteuerung beschrieben, weil ein für Stromsteuerung benötigtes Serienkreisverhalten der Varaktordiode im R-Band einfacher zu realisieren ist als ein Parallelkreisverhalten, das für eine Spannungssteuerung erforderlich wäre. Für die Dimensionierung von parametrischen Systemen ist es zweckmäßig, die Varaktordiode bei Stromsteuerung in ein ,aktives" und passives Gebiet zu unterteilen (Bild 5). Der rein passive Teil wird zu einem äußeren Netzwerk gerechnet, während die Berechnung der Mischeigenschaft der Diode direkt in einer idealen Diodenebene vorgenommen werden kann.

Mit dem vorgestellten Mikrowellenmeßaufbau ist es möglich, die Elemente der Ersatzschaltung der benutzten Varaktordiode im R-Band meßtechnisch zu bestimmen und die zur Berechnung der Mischeigenschaft wichtige Größe der dynamischen Güte zu berechnen. Außerdem läßt sich mit Hilfe von Großsignalmessungen überprüfen, wie gut die benutzte Näherung (Approximation der Aussteuerung der Diode durch eine reine Stromsteuerung) erfüllt wird.

\section{Varaktordiode}

\subsection{Allgemeines}

Die im Sperrgebiet benutzte Varaktordiode von Alpha Industries (DVE 6810F) kann im R-Band noch näherungs-

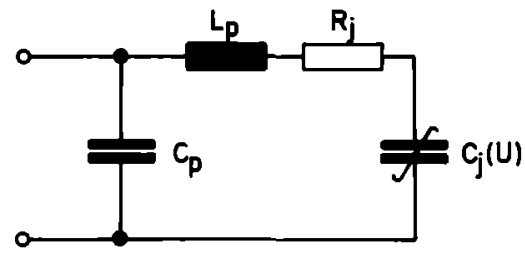

Bild 1: Ersatzschaltbild der. Varaktordiode weise durch die in [1] angegebene Ersatzschaltung (Bild 1) beschrieben werden.

$C_{\mathrm{p}}$ beschreibt die Gehäusekapazität, $L_{\mathrm{p}}$ die Zuleitungsinduktivität und $R_{\mathrm{j}}$ den Bahnwiderstand. Die Sperrschichtkapazität $C(U)$ einer Reaktanzdiode mit der Nullpunktskapazität $C(0)$ beträgt bei der Spannung $U$

$$
C(U)=\frac{C(0)}{\left(1-\frac{U}{U_{\mathrm{D}}}\right)^{n}}
$$

mit $U_{\mathrm{D}} \quad$ Diffusionsspannung,

$n$ Exponent, der vom Dotierungsprofil abhängig ist.

\subsection{Bei Stromsteuerung}

Man spricht von einer Stromsteuerung der Reaktanzdiode, wenn durch äußeren Schaltungszwang verhindert wird, daß Pumpoberwellen des Sperrschichtstromes auftreten. Dieses wird schaltungstechnisch durch einen Serienschwingkreis erreicht, der für die an der Sperrschicht erzeugten Vielfachen der Pumpfrequenz einen Leerlauf darstellen soll. Dieses Serienkreisverhalten der realen Diode läßt sich näherungsweise im mm-Wellengebiet besser realisieren als ein Parallelkreisverhalten, das für eine Spannungssteuerung erforderlich wäre. Deshalb ist es für Mischerdimensionierungen zweckmäßig, als Berechnungsmodell eine Reaktanzdiode bei Stromsteuerung zu wählen. Für eine Berechnung mit Serienkreisen ist es günstiger, statt der Sperrschichtkapazität die sogenannte Elastanz

$$
S=\frac{\mathrm{d} U}{\mathrm{~d} Q}
$$

einzuführen. Zur Bestimmung von $S$ wird (1) integriert. Man erhält

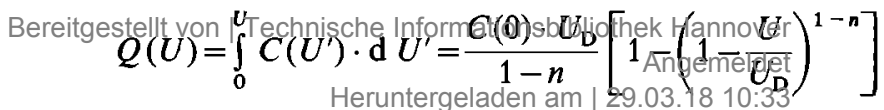


Die Integrationsgrenzen wurden dabei wie in [2] so gewählt, daß $Q(U)$ die durch äußere Spannungen verursachte Ladung ist. Den Elastanzverlauf $S(Q)$ erhält man, wenn (3) nach $U$ aufgelöst und differenziert wird:

$$
S(Q)=\frac{1}{C(0)}\left[1-(1-n) \frac{Q}{C(0) \cdot U_{\mathrm{D}}}\right]^{\frac{n}{1-n}} .
$$

Wird die Diode in der Umgebung einer festen Sperrspannung $U_{\mathrm{v}}^{\prime}$ mit einem sinusförmigen Pumpstrom durchgesteuert, dann erhält man die in Bild 2 gestrichelt gezeichnete harmonische Pumpladungsaussteuerung $Q\left(\omega_{\mathrm{p}} t\right)$ $=Q_{\mathrm{p}} \cos \left(\omega_{\mathrm{p}} t+\psi_{\mathrm{p}}\right)$, die eine verzerrte Ausgangsspannung zur Folge hat. Die in der verzerrten Ausgangsspannung enthaltene Richtspannung bewirkt nach [2] so lange eine Arbeitspunktverschiebung, bis die Ausgangsspannung bezogen auf die ursprüngliche, durch $U_{v}^{\prime}$ festgelegte Bezugsachse keine Richtspannung mehr enthält. Der Arbeitspunkt $Q_{v}^{\prime}$ wandert bis an die Stelle $Q_{v}$, wo eine Flächengleichheit zwischen den Halbwellen der Ausgangsspannung (bezogen auf $U_{v}^{\prime}$ ) erreicht ist. Eine geschlossene Berechnung des neuen Arbeitspunktes $Q_{v}$ ist nur für den Sonderfall möglich, daß in (1) der Exponent den Wert $n$ $=0,5$ (abrupte Dotierung) annimmt.

\section{Diodenmeßverfahren}

\subsection{Bestimmung der Diodenelemente}

Bei der Auswahl eines geeigneten Diodenmeßverfahrens wurde darauf geachtet, daß die Diodenmessungen bei der Frequenz und in dem Diodenhalter ausgeführt werden konnten, die später auch im Betriebsfall vorlagen, um Frequenz- und Diodenhaltereinflüsse auszuschließen. Deshalb wurde auf Diodenmeßverfahren verzichtet, die bei tiefen Frequenzen oder mit einem speziellen Aufbau (Resonator, Stempel) durchgeführt werden mußten. Einige Verfahren berechnen aus dem gemessenen Reflexionsfaktorverlauf die Elemente der Diodenersatzschaltung, indem ein umfangreiches Gleichungssystem gelöst oder die GröBe der Elemente so lange variiert werden muß, bis der gemessene mit dem berechneten Verlauf übereinstimmt. Diese Verfahren sind mit einem großen Fehler behaftet, besonders bei der Bestimmung des Bahnwiderstandes $R_{\mathrm{j}}$, so $\mathrm{da} B$ sie nicht in Betracht kommen.

Für Reaktanzdioden, die bei ihrer Serienresonanzfrequenz gemessen werden können, eignet sich am besten das Transmissionsverfahren [3]. In der Nähe der Serienresonanzfrequenz wird die Diode durch einen Serienschwingkreis beschrieben. Die Elemente $L_{\mathrm{p}}$ und $R_{\mathrm{j}}$ werden als konstant, die Sperrschichtkapazität nur als Funktion der

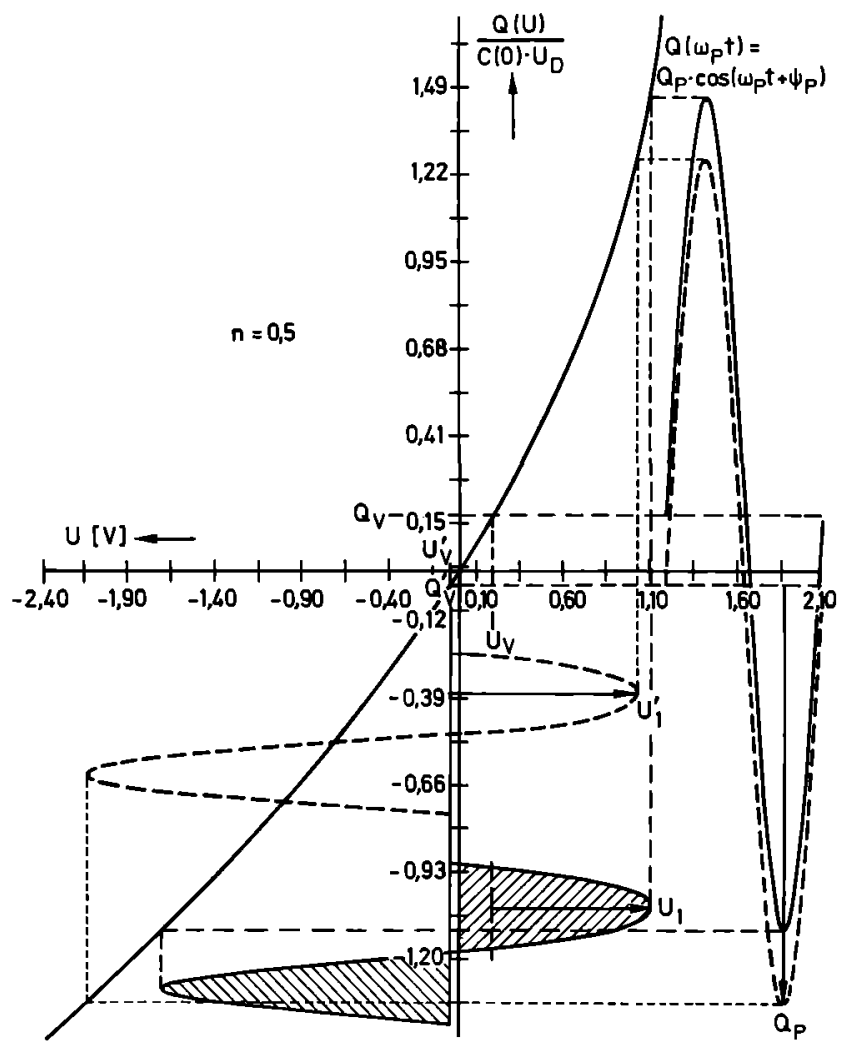

Bild 2: Aussteuerung der $Q(U)$-Kennlinie einer Varaktordiode bei Stromsteuerung

angelegten Gleichspannung angenommen. Die Diode wird in der Mitte eines auf die Diodenhöhe reduzierten Hohlleiters eingebaut, so da $B$ die eingebaute Diode mit dem elektrischen Modell in Bild 3 beschreibbar ist. Die Ebenen 1 $-1^{\prime}$ und $5-5^{\prime}$ sind für eine Messung zugänglich. Der kontinuierliche Hohlleiterübergang (Taper) wird so konzipiert, da $B$ der Eigenreflexionsfaktor des Tapers vernachlässigbar ist.

Durch die Reduzierung der Hohlleiterhöhe wird der sogenannte Leitungswellenwiderstand $Z_{\mathrm{oL}}$ verkleinert, während der Feldwellenwiderstand $Z_{\mathrm{oF}}=\left(\lambda_{\mathrm{H}} / \lambda_{c}\right) 377 \Omega$ unabhängig von der Hohlleiterhöhe ist. Da im Hohlleiter in axialer Richtung eine Feldkomponente existiert, ist es schwierig, einen eindeutigen Leitungswellenwiderstand für die $\mathrm{H}_{10}$-Welle zu definieren. In [4] werden drei Leitungswellenwiderstände $Z_{\mathrm{oL}}(U, I), Z_{\mathrm{oL}}(P, U)$ und $Z_{\mathrm{oL}}(P, I)$ aus

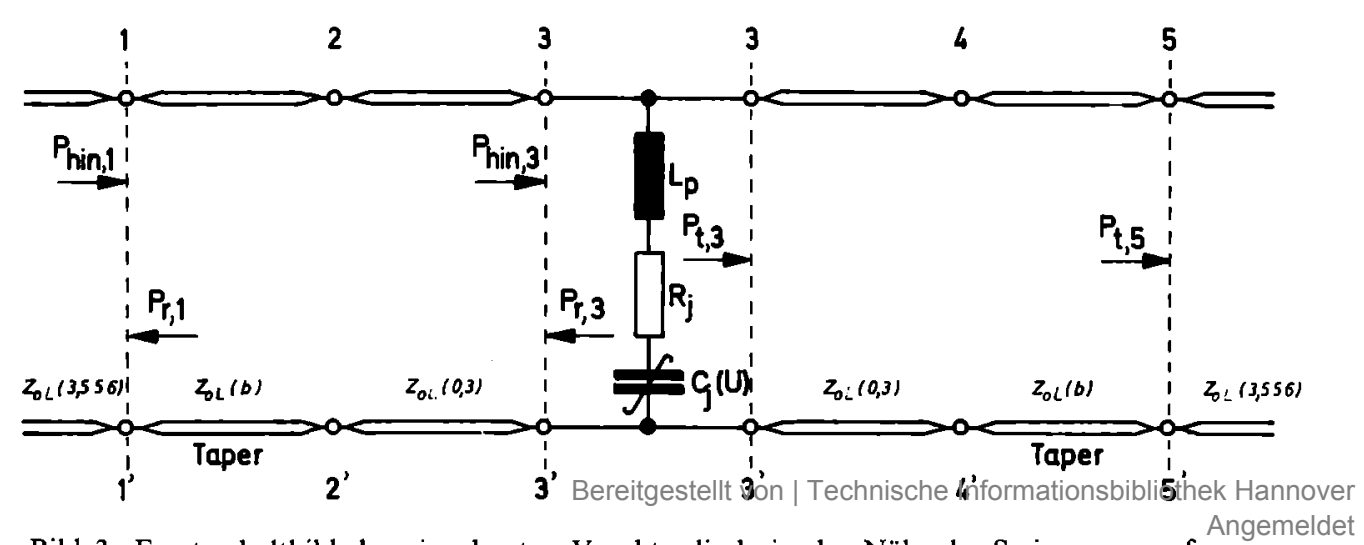

Bild 3: Ersatzschaltbild der eingebauten Varaktordiode in der Nähe der Serienzesgnanzfrequegz 0:33 


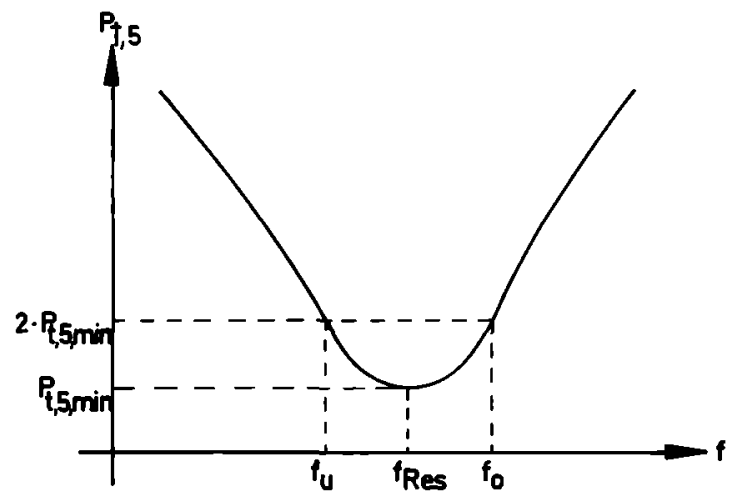

Bild 4: $P(f)$-Kennlinie eines Serienschwingkreises

den Größen Spannung, Strom und Leistung abgeleitet, die sich im Zahlenwert erheblich unterscheiden. Für den vorliegenden Fall des Einbaus der Diode, mit dem kleinen Durchmesser von etwa $0,8 \mathrm{~mm}$ in den $a=7,112 \mathrm{~mm}$ breiten und $b=0,3 \mathrm{~mm}$ hohen Hohlleiter $\left(b \ll \lambda_{\mathbf{H}} / 4\right)$, gelten die in [4] getroffenen Voraussetzungen für die Benutzung des aus Leistung und Spannung abgeleiteten Leitungswellenwiderstandes

$$
Z_{\mathrm{oL}}(P, U)=\frac{b}{a} \cdot \frac{\lambda_{\mathrm{H}}}{\lambda_{c}} 754 \Omega
$$

mit

$$
\begin{aligned}
& \lambda_{c}=\frac{c}{f}, \quad(c \text { Lichtgeschwindigkeit }), \\
& \lambda_{\mathrm{H}}=\frac{\lambda_{c}}{\sqrt{1-\left(\frac{\lambda_{c}}{2 a}\right)^{2}}} .
\end{aligned}
$$

Die hinlaufende Welle mit dem Leistungsinhalt $P_{\text {hin, } 1}$ in Bild 3 wird durch die erste Taperhälfte $(1-2)$ und das 2,5 mm lange, 0,3 mm hohe Hohlleiterstück (2-3) um den Faktor $D$ auf den Wert $P_{\text {hin, }}$ bedämpft. Durch den Serienschwingkreis in der Ebene 3-3' wird der Anteil $\boldsymbol{P}_{\mathrm{r}, 3}$ der einfallenden Leistung $P_{\text {hin, } 3}$ reflektiert, während die transformierte Leistung $P_{\mathrm{l}, 3}$, wieder um den Faktor $D$ bedämpft, am reflexionsfrei abgeschlossenen Tor (Ebene 5 $-5^{\prime}$ ) als $P_{1,5}$ gemessen werden kann.

Bei der folgenden Berechnung werden die an der Störstelle $3-3^{\prime}$ angeregten höheren Hohlleitermoden, die wegen der Hohlleiterabmessungen nicht ausbreitungsfähig sind und deshalb exponentiell abklingen, vernachlässigt.

Für den Resonanzfall $f=f_{\text {Res }}$ wird die minimale Ausgangsleistung $P_{\mathrm{t}, \mathrm{s}, \min }$ gemessen. Mit der Beziehung

$$
T=\frac{P_{\mathrm{hin}, 3}}{P_{\mathrm{l}, 3, \min }}=D^{2} \frac{P_{\mathrm{hin}, 1}}{P_{\mathrm{l}, 5, \text { min }}}
$$

berechnet sich der Bahnwiderstand zu

$$
R_{\mathrm{j}}=\frac{Z_{\mathrm{oL}}(0,3)}{2} \frac{1}{\sqrt{T}-1} .
$$

Variiert man die Frequenz bis zu den Frequenzen $f_{\mathrm{u}}$ und $f_{\mathrm{o}}$ in Bild 4, wo die doppelte Ausgangsleistung $\left(2 P_{1,5, \min }\right)$ auftritt, dann lassen sich die Elemente $C_{\mathrm{j}}(U)$ und $L_{\mathrm{p}}$ mit Hilfe der Gleichungen

$$
C_{\mathrm{j}}(U)=\frac{\sqrt{T}-1}{\pi Z_{\mathrm{oL}}(0,3)} \sqrt{1-\frac{2}{T}} \frac{f_{\mathrm{o}}-f_{\mathrm{u}}}{f_{\mathrm{o}} f_{\mathrm{u}}}
$$

und

$$
L_{\mathrm{p}}=\frac{1}{4 \pi^{2}} \frac{1}{f_{\mathrm{o}} f_{\mathrm{u}} C_{\mathrm{j}}(U)}
$$

bestimmen. Bei Dioden mit niedriger Güte ist die 3-dBBandbreite $f_{\mathrm{o}}-f_{\mathrm{u}}$ ziemlich groß und (8) und (9) dürfen nicht mehr in dieser Form verwendet werden, da der Leitungswellenwiderstand $Z_{\mathrm{oL}}(0,3)$ aus (5) frequenzabhängig und die benutzte Näherung

$$
\left.\left.\left.Z_{\mathrm{oL}}(0,3)\right|_{f_{\mathrm{u}}} \approx Z_{\mathrm{oL}}(0,3)\right|_{f_{\mathrm{Rea}}} \approx Z_{\mathrm{oL}}(0,3)\right|_{f_{\mathrm{o}}}
$$

nicht mehr zulässig ist. Die für diesen Fall erforderlichen Gleichungen sind ebenfalls in [3] angegeben.

Die Gehäusekapazität $C_{\mathrm{p}}$ in Bild 1 kann mit Hille einer Reflexionsmessung (Betrag und Phase) bestimmt werden. Bei der Frequenz $f_{1}$ wird durch Variation der Liodenvorspannung Serienresonanzverhalten $\left(P_{\mathrm{t}, \mathrm{min}}\right)$ eingestellt.

Mit der gefundenen Vorspannung für Serienresonanz bei $f_{1}$ wird bei der Frequenz $f_{2}$ der Reflexionsfaktor nach Betrag und Phase am Anfang des. Tapers (Ebene 1-1' in Bild 3) gemessen. Das andere Tor des Tapers ist wieder reflexionsfrei abgeschlossen, so da $\beta$ der parallel zur Diode liegende Widerstand mit (5) berechnet werden kann. Mit dem Dämpfungsfaktor $D$ und der elektrischen Länge $l_{\mathrm{T}} / 2$ (Ebene 1 bis Ebene 3 in Bild 3) läßt sich dann der Reflexionsfaktor $\underline{r}_{3-3^{\prime}}$ in der Ebene $3-3^{\prime}$ des Bildes 3 berechnen, und damit ergibt sich die gesuchte Gehäusekapazität $\mathrm{zu}$

$$
C_{\mathrm{p}}=\frac{1}{\mathrm{j} \omega Z_{\mathrm{oL}}(0,3)}\left[\frac{1-\underline{r}_{3-3^{\prime}}}{1+\underline{r}_{3-3^{\prime}}}-\frac{Z_{\mathrm{oL}}(0,3)}{R_{\mathrm{j}}+\mathrm{j}\left(\omega L_{\mathrm{p}}-\frac{1}{\omega C_{\mathrm{j}}(U)}\right)}-1\right]
$$

Ein zusätzlicher Imaginärteil für $C_{\mathrm{p}}$ in (10), der auf Grund der Meßfehler entsteht, gibt Aufschluß über die Genauigkeit der Messung.

\subsection{Bestimmung des $C(U)$-Exponenten $n$ und der dynami- schen Güte $q$}

Wird die Serieninduktivität $L_{\mathrm{p}}$ als konstant angenommen, dann kann das MeBverfahren [3] abgeändert werden, um schnell den Kennlinienverlauf $C_{\mathrm{j}}(U)$ zu erhalten. Für verschiedene Vorspannungen wird die Serienresonanzfrequenz $f_{\text {Res }}$ gemessen $\left(P_{1}, 5\right.$, min $)$ und aus der Resonanzbeziehung

$$
C_{\mathrm{j}}(U)=\frac{1}{\omega_{\text {Res }}^{2} L_{\mathrm{p}}}
$$

der Wert der Sperrschichtkapazität für die eingestellte Vorspannung ermittelt. Die gemessene $C(U)$-Kennlinie kann mit Hilfe eines Computerprogramms mit der theoretischen $C(U)$-Kennlinie aus (1) verglichen werden. Der Exponent $n$ in (1) wird so lange variiert, bis die größte Übereinstimmung $\mathrm{zwischen}$ beiden Kennlinien vorliegt.

Die Sperrschichtkapazität $C(U)$ wird mit Hilfe der Elastanz $S(Q)$ dargestellt, welche in die zwei Anteile $S^{(0)}$ und $S^{(1)}\left(\right.$ Bild 5) aufgeteilt wird. $1 / S^{(0)}$ ist eine mittlere Kapazität; diese hängt i.a. von der Vorspannung $U_{\mathrm{v}}$, der Pumpamplitude $Q_{\mathrm{p}}$ und der Kennlinienform der Diode ab. Der reziproke Wert der Elastanz $\underline{S}^{(1)}$ hat dagegen eine andere physikalische Bedeutung als die Elastanz $S^{(0)} ; \underline{S}^{(1)}$ beschreibt eine Leistungsumsetzung zwischen den Signalen bei den auftretenden Frequenzen Die weiteren Verknüpfungsgrößen $S^{(\eta+1)}(\eta=1,2,3 \ldots)$ kännen vernachlässigt werden. Die Varaktorersatzschaltung in 8 ild 53 wird in ei- 


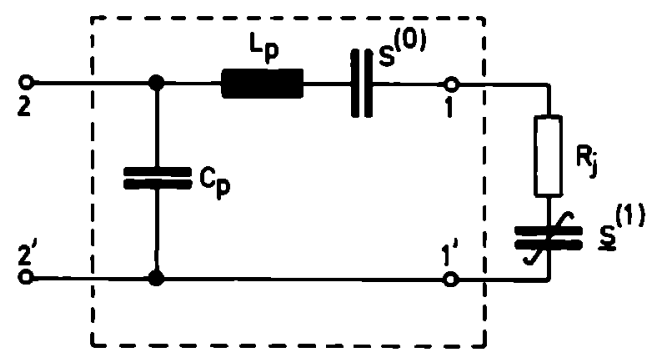

Bild 5: Ersatzschaltbild der Varaktordiode für Stromsteuerung $\boldsymbol{R}_{\mathrm{j}} \quad$ Bahnwiderstand

$\begin{array}{ll}L_{\mathrm{p}} & \text { Zuleitungsinduktivität } \\ \boldsymbol{C}_{\mathrm{p}} & \text { Gehäusekapazität }\end{array}$

nen verlustlosen Vierpol $\left(C_{\mathrm{p}}, L_{\mathrm{p}}, S^{(0)}\right)$ und einen Zweipol $\left(R_{\mathrm{j}}, \underline{S}^{(1)}\right)$ aufgeteilt. Der Zweipol bewirkt mit der Elastanz $S^{(1)}$ einen aktiven oder passiven Betrieb; der mit enthaltene Bahnwiderstand $\boldsymbol{R}_{\mathrm{j}}$ beschreibt die Verluste im Halbleitermaterial. Eine Möglichkeit zur Beschreibung des Zweipols bietet die sogenannte dynamische Güte $q$, die definiert ist als

$$
q=\frac{\left|S^{(1)}\right|}{\omega R_{\mathrm{j}}}, \quad(\omega \text { Signalkreisfrequenz) }
$$

Für eine harmonische Pumpaussteuerung läßt sich (4) schreiben als

$S\left(\omega_{\mathrm{p}} t\right)=\frac{1}{C(0)}\left[1-(1-n) \frac{Q_{\mathrm{v}}+Q_{\mathrm{p}} \cos \left(\omega_{\mathrm{p}} t+\psi_{\mathrm{p}}\right)}{C(0) U_{\mathrm{D}}}\right]^{\frac{n}{1-n}}$.

Für (13) kann eine Fourierentwicklung durchgeführt werden, weil der zeitvariable Arbeitspunkt sich periodisch mit der Frequenz $f_{\mathrm{p}}$ um den festen Arbeitspunkt $Q_{\mathrm{v}}$ ändert.

Die Funktion $S\left(\omega_{\mathrm{p}} t\right)$ in (13) wird durch die komplexe Fourierreihe

$$
S\left(\omega_{\mathrm{p}} t\right)=S^{(0)}+\sum_{\eta=1}^{\infty}\left(S^{(\eta)} \mathrm{e}^{\mathrm{j} \eta \omega_{\mathrm{p}} t}+\underline{S}^{(\eta) *} \mathrm{e}^{-\mathrm{j} \eta \omega_{\mathrm{p}} t}\right)
$$

mit den Koeffizienten

$$
\underline{S}^{(\eta)}=\frac{\mathrm{e}^{\mathrm{j} \eta \psi_{\mathrm{p}}}}{2 \pi} \int_{0}^{2 \pi} S\left(\omega_{\mathrm{p}} t\right) \mathrm{e}^{-\mathrm{j} \eta\left(\omega_{\mathrm{p}} t+\psi_{\mathrm{p}}\right)} \mathrm{d}\left(\omega_{\mathrm{p}} t+\psi_{\mathrm{p}}\right)
$$

dargestellt; dabei gilt $\underline{S}^{(-\eta)}=\underline{S}^{(\eta) *}$, da $S\left(\omega_{\mathrm{p}} t\right)$ eine reelle Funktion ist. Mit einem fertigen Programm des regionalen Rechenzentrums Hannover wurden die Fourierkoeffizienten $S^{(0)}, \underline{S}^{(1)}$ und $\underline{S}^{(2)}$ aus (15) berechnet. Die Berechnung der Größe $\underline{S}^{(2)}$ wird zur Kontrolle durchgeführt, ob die Vernachlässigung der höheren Fourierkoeffizienten gerechtfertigt ist.
Die Aussteuerung wird so vorgenommen, da $\beta$ der Richtstrom in der Größenordnung von einigen nA liegt, um nur die unvermeidlichen Verluste des Bahnwiderstandes berücksichtigen zu müssen. Für die feste Frequenz $f_{\mathrm{p}}$ wird für verschiedene Eingangsleistungen $P_{\text {hin, } 1}$ in Bild 3 mit der Variation der Vorspannung Serienresonanzverhalten $\left(P_{\mathrm{L}, 5, \mathrm{~min}}\right)$ eingestellt. Die im Bahnwiderstand umgesetzte Leistung $P_{\mathrm{j}}$ berechnet sich aus

$$
P_{\mathrm{j}}=P_{\text {hin, } 3}-P_{\mathrm{r}, 3}-P_{\mathrm{t}, 3},
$$

wenn die Verluste der höheren Moden vernachlässigt werden. Bei Annahme einer reinen Stromsteuerung ergibt sich der Strom durch die Diode aus der Beziehung

$$
I_{\mathrm{j}}=\sqrt{\frac{2 P_{\mathrm{j}}}{R_{\mathrm{j}}}} .
$$

Gleichzeitig wird der Strom $I_{\mathrm{j}}$ mit Hilfe eines Iterationsprogramms aus der Darstellung in Bild 2 berechnet:

Als feste Größen werden die Vorspannung $U_{\mathrm{v}}^{\prime}$ und der Richtstrom vorgegeben. Die Iteration besteht darin, bei angenommener sinusförmiger Pumpaussteuerung die neue Vorspannung $U_{\mathrm{v}}$ und die Amplitude $U_{1}$ so lange zu variieren, bis die Flächengleichheit um $U_{v}^{\prime}$ und der angegebene Richtstrom vorliegen. Dann kann aus der Pumpamplitude $Q_{\mathrm{p}}$ mit

$$
I_{\mathrm{j}}=\omega Q_{\mathrm{p}}
$$

der Sperrschichtstrom $I_{\mathrm{j}}$ bestimmt und mit (17) verglichen werden.

\section{Entwicklung der Schaltungskomponenten}

\subsection{Kontinuierlicher Hohlleiterübergang (Taper)}

Die kontinuierliche Transformation wird näherungsweise mit einer verlustlosen, inhomogenen Leitung durchgeführt, deren Wellenwiderstand $Z_{0}(z)$ eine Funktion der Ortskoordinate $z$ ist, während die Phasenkonstante $\beta$ unabhängig von $z$ angenommen wird [5]. In [5-10] sind mehrere Berechnungswege, Optimierungen und praktische Realisierungen angegeben. Aus den dort angegebenen Verläufen wurde die Funktion

$$
\frac{Z_{0}(z)}{Z_{0}(z=0)}=\left[\frac{Z_{0}(z=l)}{Z_{0}(z=0)}\right] \frac{z}{l}\left[1-\frac{\sin \left(2 \pi-\frac{z}{l}\right)}{2 \pi-\frac{2}{l}}\right]
$$

\section{( $l$ Länge einer Taperhälfte)}

ausgewählt, die am Anfang der Leitung $(z=0)$ einen Reflexionsfaktor von

$$
r(z=0)=\frac{1}{2} \ln \left[\frac{Z_{0}(z=l)}{Z_{0}(z=0)}\right] \frac{1}{1-\left(\frac{2 l}{\lambda_{\mathrm{H}}}\right)^{2}} \frac{\sin \left(2 \pi \frac{l}{\lambda_{\mathrm{H}}}\right)}{2 \pi \frac{l}{\lambda_{\mathrm{H}}}} \mathrm{e}^{-\mathrm{j} 2 \pi \frac{l}{\lambda_{\mathrm{H}}}}
$$

\subsection{Bestimmung der Pumpamplitude bei Stromsteuerung}

Die Berechnung der Amplitude $U_{1}$ der rechten Spannungshalbwelle in Bild 2 ist mit einer Richtstrommessung möglich. Dafür muß die statische $I(U)$-Kennlinie aufgenommen werden. Mit Hilfe eines Polynoms 4. Grades ließ sich die $I(U)$-Kennlinie im benötigten Bereich approximieren, so daß der Richstrom als Funktion der Vorspan nung und der Spannungsamplitude aus einer Fourieranalyse als Gleichanteil bestimmt werden konnte. ergibt. In [11] wird gezeigt, daß der Wellenwiderstand $Z_{0}(z)$ proportional zur Hohlleiterhöhe $b(z)$ ist. Damit erhält man aus (19)

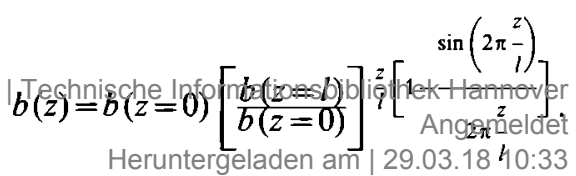


Die Taperfunktion (21) wurde in einem Stück $(3,556 \mathrm{~mm}$ auf $0,3 \mathrm{~mm} ; 5 \mathrm{~mm}$ langer, für den Diodeneinbau vorgesehener, $0,3-\mathrm{mm}$-Hohlleiter; $0,3 \mathrm{~mm}$ auf $3,556 \mathrm{~mm})$ gefräst. Hierzu wurden im Abstand von $0,01 \mathrm{~mm}$ die Werte der Funktion (21) als Treppe vorgefräst und dann geschliffen. Das Aufbringen der Silberschicht und der Zusammenbau geschahen in gleicher Weise wie bei der Herstellung der Hohlleiterfilter in [12]. Die Messungen ergaben bei der Betriebsfrequenz $f_{\mathrm{p}}=31 \mathrm{GHz}$ einen Reflexionsfaktorbetrag von $0,05 \%$ und im benötigten Frequenzband von $27,5 \mathrm{GHz}$ bis $34,5 \mathrm{GHz}$ einen maximalen Reflexionsfaktorbetrag von $3 \%$. Die Einfügungsdämpfung des gesamten UUberganges (2 Taperhälften mit je $l=25 \mathrm{~mm}$ Länge und ein $5 \mathrm{~mm}$ langer, für den Diodeneinbau vorgesehener, $0,3 \mathrm{~mm}$ hoher Hohlleiter) betrug $0,3 \mathrm{~dB}$.

\subsection{Koaxiales Filter}

Das koaxiale Filter wird benötigt, um der Diode eine Gleichspannung zuführen zu können. Damit die Ersatzschaltung in Bild 3 gilt, muß das Filter im benutzten Frequenzband von $27,5 \mathrm{GHz}$ bis $34,5 \mathrm{GHz}$ ein fast ideales Sperrverhalten aufweisen. Das koaxiale Tiefpaßfilter wurde aus Leitungsstücken mit unterschiedlichem Wellenwiderstand aufgebaut. Für die Berechnung wurde zuerst ein Modell aus konzentrierten Elementen nach [13] gewählt. Es zeigte sich, daß die Berechnung nur für sehr dünne, kapazitive Scheiben gilt. Besseres Sperrverhalten läßt sich aber mit einem Filter (Bild 6) erzielen, wenn die Leitungslängen dicht bei $\lambda_{\text {erf }} / 4$ liegen.

Die Durchmesser in Bild 6 wurden so gewählt, daß nach [14] bis $35 \mathrm{GHz}$ nur eine reine TEM-Wellenausbreitung möglich ist. Für die Anordnung in Bild 6 wurde ein einfaches Ersatzschaltbild aus Leitungsstücken mit unterschiedlichem Wellenwiderstand entwickelt (Bild 7). Der Wellenwiderstand $Z_{0}$ einer verlustlosen Koaxialleitung mit TEM-Wellenausbreitung ist nach [13]

$$
Z_{0}=\ln \left(\frac{d_{\mathrm{a}}}{d_{\mathrm{i}}}\right) \frac{60 \Omega}{\sqrt{\varepsilon_{\mathrm{r}}}}
$$

mit $d_{\mathrm{a}}=$ Durchmesser des Außenleiters,

$d_{\mathrm{i}}=$ Durchmesser des Innenleiters.
Das Leitungsstück der Länge $l_{1}$ mit dem niedrigen Wellenwiderstand $Z_{0,1}$ in Bild 7 beschreibt die kapazitive Scheibe mit dem Dielektrikum $\varepsilon_{r}$, während der dünne Innenleiter in Bild 6 durch das Leitungsstück der Länge $l_{\mathrm{h}}$ mit dem hohen Wellenwiderstand $Z_{\mathrm{o}, \mathrm{h}}$ beschrieben wird. Für die Diskontinuität (Scheibe - dünner Innenleiter) wird eine Sprungkapazität $C_{\text {spr }}$ nach [15] eingeführt. Die Ersatzschaltung in Bild 7 wurde mit der Leitungstheorie berechnet. Die Ableitung der Berechnung, das Computeroptimierungsprogramm sowie mehrere Kurven des Phasen- und Dämpfungsverlaufes sind in [16] angegeben. Für eine Abschlußimpedanz $\underline{Z}$ mit beliebigem Imaginärteil erhält man für $\operatorname{Re}\{\underline{Z}\}=1 \Omega$ eine theoretische Sperrdämpfung $\left|a_{\mathrm{sp}}\right|$ $=83 \mathrm{~dB}$ und für $\operatorname{Re}\{\underline{Z}\}=50 \Omega$ ein $\left|a_{\text {sp }}\right|=100 \mathrm{~dB}$, während die Phase des Eingangsreflexionsfaktors in beiden Fällen $\arg \left\{r_{1-1^{\prime}}\right\}=-179,9976^{\circ}$ erreicht. Da im R-Band keine koaxialen Messungen durchgeführt werden konnten, wurden zwei Filtermodelle bei $f_{1}=8 \mathrm{GHz}$ und $f_{2}=17 \mathrm{GHz}$ mit den gleichen Charakteristiken wie bei $f_{\mathrm{p}}=31 \mathrm{GHz}$ aufgebaut und gemessen. Die Phasenmessungen $\left(\arg \left\{r_{1-1}\right\} \approx 180^{\circ}\right)$ erfüllten sehr gut die Theorie. Wegen der großen Sperrdämpfung konnte der Dämpfungsverlauf im Sperrgebiet nur an Modellen mit zwei und drei kapazitiven Scheiben gemessen werden. Auch hier entsprachen die Messungen den theoretischen Erwartungen. Deshalb kann auch mit sehr großer Wahrscheinlichkeit angenommen werden, da B im R-Band bis $35 \mathrm{GHz}$ der auftretende Sperrdämpfungs- und Phasenverlauf den Berechnungen entspricht. Indirekte Messungen mit eingebauter Diode (s. Abschnitt 5) bestätigen diese Annahme.

\subsection{Diodeneinbau}

Die in Bild 8 skizzierte Diode besteht aus der Keramikschicht Tb und den beiden Metallteilen Ta und Tc. Damit die Diode leichter eingebaut werden kann, wurde vom Hersteller an die Metallschicht Tc der Metallstempel Td$\mathrm{Te}$ angeschweißt. Dadurch entstand eine starke Exzentrizität zwischen den Metallanschlüssen Ta und Td-Te.

Für die Diodenhalterung wurde am koaxialen Anschlußflansch des Tores B eine Hülse mit Innengewinde befestigt, die als Zentrierhalter dient. In diese Hülse läßt sich nun die eigentliche Diodenzange einschrauben. Die

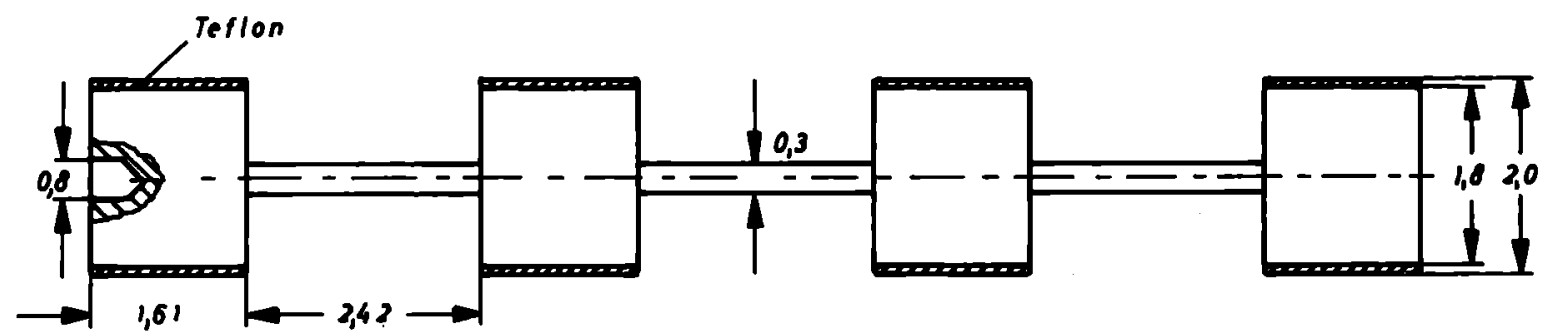

Bild 6: Koaxiales Tiefpaßfilter

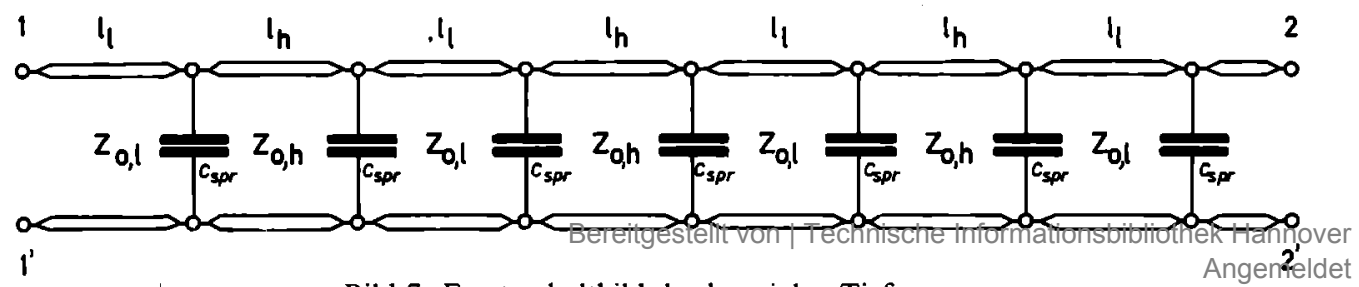

Bild 7: Ersatzschaltbild des koaxialen Tiefpassesfergeladen am | 29.03.18 10:33 
Diode wird mit dem Metallstempel Te in eine geschlitzte Scheibe eingesetzt, die sich wegen ihrer konischen Ausführung mit Hilfe eines eingeschraubten Dorns öffnen und schließen läßt. Die Schlitze in der Scheibe würden das Feldbild des Hohlleiters stören. Deshalb wird eine gemäß Bild 8 versilberte Abdeckkappe Tf auf die Scheibe gesetzt, die mit dem reduzierten Hohlleiter abschließt. Zentriert wird die Diode mit dem Metallteil Ta, das sich in die Bohrung der Tiefpaßscheibe einfädelt, wenn die Diodenzange in die Hülse geschraubt wird. Die Exzentrizität zwischen dem Metallteil Ta und dem Stempel Te wird durch die Federwirkung der geschlitzten Scheibe ausgeglichen. Mit der Dicke des versilberten Plättchens Tg lassen sich Längenänderungen der Teile $\mathrm{Ta}$ und $\mathrm{Tb}$ so ausgleichen, $\mathrm{daB}$ sich nur die Keramikschicht $\mathrm{Tb}$ im Hohlleiter befindet. Die Halterung wurde auch zum Einbau der Abstimmvaraktordiode in [16] benutzt. Dort sind die Konstruktionszeichnungen der gesamten Diodenhalterung (Zange, Zentrierstück, Dorn) wiedergegeben.

\section{Diodenmessungen}

Der für die Diodenmessungen benötigte Meßaufbau ist in Bild 9 skizziert. Die eingezeichnete Meßebene wurde für den gesamten Frequenzbereich 27,5 bis $34,5 \mathrm{GHz}$ kalibiert, so da $B$ aus einer $P_{\text {hin }}$ - und $P_{\mathrm{r}}$-Leistungsmessung exakt die Leistungen $P_{\mathrm{hin}, 1}$ und $P_{\mathrm{r}, 1}$ in der Meßebene berechnet werden konnten. Ebenfalls wurde eine Kalibrierung für die Leistungen $P_{\mathrm{t}, 5}$ und $P_{\mathrm{t}}$ durchgeführt.

Bevor mit den Meßverfahren nach Abschnitt 3 gearbeitet werden konnte, mußte untersucht werden, ob die elektrische Ersatzschaltung nach Bild 3 den tatsächlichen Meßaufbau beschreibt.

In den Taper wurden die Öffnungen für die Koaxialleitung und die Diodenhalterung gebohrt und anschließend mit versilberten Metallstempeln wieder verschlossen, um zu prüfen, ob durch den notwendigen Auseinander- und Zusammenbau keine Spalten oder Verformungen entstanden sind. Danach geschah der Einbau der Diodenhalterung mit Diode, die aber nicht wie in Bild 8 mit dem Metallanschluß Ta in die Scheibe des Tiefpasses gesteckt wurde, sondern in die 0,8-mm-Öffnung eines versilberten Metallstempels. Eine Gleichspannung kann bei diesem Aufbau der Diode nicht zugeführt werden; deshalb wurden die Messungen mehrerer Dioden nach [3] bei $U=0 \mathrm{~V}$ durchgeführt.

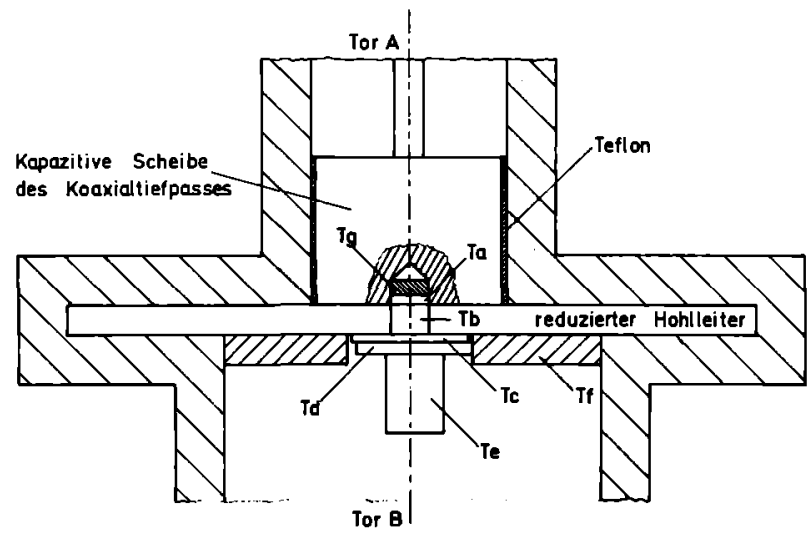

Bild 8: Diodeneinbau

Anschließend wurde der Metallstempel entfernt und dafür das koaxiale Tiefpaßfilter (Bild 6) eingebaut. Beide Messungen brachten die gleichen Ergebnisse, so daB damit das berechnete Kurzschlußverhalten des Tiefpasses $\left(\arg \left\{r_{1-1}\right\} \approx 180^{\circ}\right)$ indirekt bestätigt wurde.

Mehrere Dioden wurden bei verschiedenen Vorspannungen nach [3] ausgemessen $\left(P_{\text {hin }, 1}=20 \mu \mathrm{W}\right.$, s. Bild 9). Dabei schwankte die berechnete Serieninduktivität $L_{\mathrm{p}}$ maximal um $2 \%$ um den vom Hersteller angegebenen Wert $L_{\mathrm{p}}$ $=0,16 \mathrm{nH}$, während die Sperrschichtkapazität $C_{\mathbf{j}}(U$ $=0 \mathrm{~V}$ ) im Durchschnitt um $15 \%$ höher lag als der im Datenblatt angegebene Wert. Die Bahnwiderstände lagen bei $R_{\mathrm{j}} \approx 2 \Omega$. Aus dem Datenblatt lassen sich mit Hilfe der angegebenen Grenzfrequenz Bahnwiderstände von etwa $R_{\mathrm{j}} \approx 1,8 \Omega$ berechnen.

Falls die Serienresonanzfrequenz der Diode nur unwesentlich aus dem Meßbereich herausfällt, ist es mit einer Verschiebung der Kurzschlußebene des Tiefpasses möglich, durch Transformation mit einer zusätzlichen Serienreaktanz die Resonanzfrequenz in den gewünschten Frequenzbereich zu ziehen. Dafür ist ein zusätzlicher Innenleiter erforderlich, der zwecks Zentrierung am besten durch Teflon gehalten wird. Der Innenleiter muß so dimensioniert werden, daß eine Öffnung vorhanden ist, um den Metallanschluß Ta der Diode aufnehmen zu können.

Für vier Dioden wurde die Sperrschichtkapazität $C_{\mathrm{j}}$ als Funktion der angelegten Vorspannung aus den Messungen

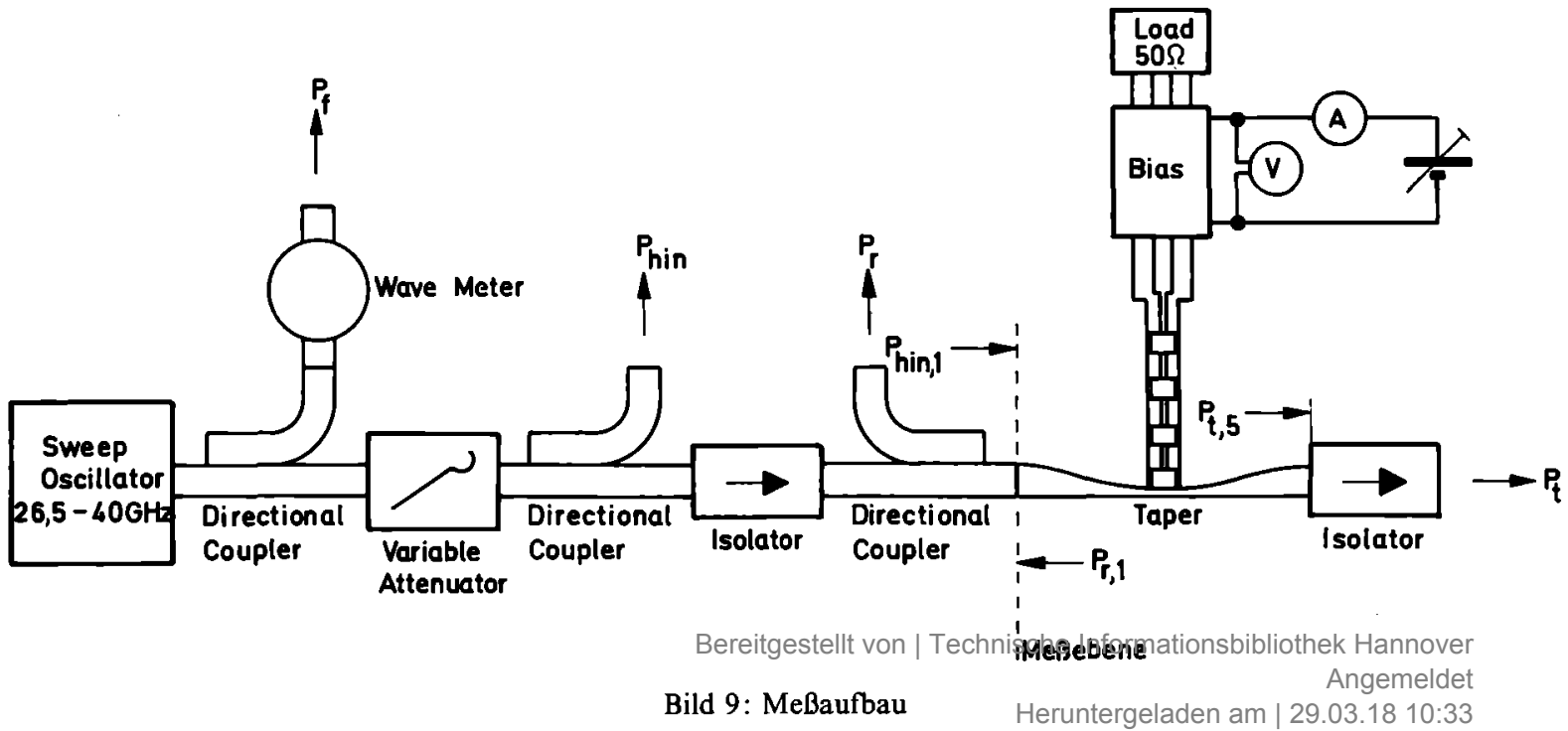


mit (11) bestimmt. Der Exponent $n$ in (1) wurde so lange variiert, bis die größte Übereinstimmung zwischen der gemessenen und berechneten $C_{\mathrm{j}}(U)$-Kennlinie auftrat. Dabei zeigte sich auch, daß die vom Hersteller angegebene Diffusionsspannung $U_{\mathrm{D}}=1,2 \mathrm{~V}$ den tatsächlichen Verlauf gut beschreibt. Die Exponenten der gemessenen Dioden lagen bei $n \approx 0,3$.

Für eine Diode sollen die Meßergebnisse ausführlich beschrieben werden: Mit dem Meßverfahren [3] und den Gleichungen (6) bis (9) wurden die Werte

$$
\begin{gathered}
R_{\mathrm{j}}=2,1 \Omega \\
C_{\mathrm{j}}(0 \mathrm{~V})=0,1585 \mathrm{pF} \\
L_{\mathrm{p}}=0,16 \mathrm{nH}
\end{gathered}
$$

ermittelt. Mit (11) ließ sich die $C_{\mathrm{j}}(U)$-Kennlinie im Eindeutigkeitsbereich des koaxialen Tiefpasses ermitteln. Ein Rechnerprogramm zur Beschreibung der gemessenen $C_{\mathrm{j}}(U)$-Kennlinie mit (1) ergab

$$
\begin{gathered}
n=0,31, \\
U_{\mathrm{D}}=1,2 \mathrm{~V} .
\end{gathered}
$$

Bei den Frequenzen $f_{1}=27,5 \mathrm{GHz}$ und $f_{1}^{\prime}=28 \mathrm{GHz}$ wurde mit dem Meßaufbau in Bild $9\left(P_{\text {hin, } 1}=1 \mathrm{~mW}\right)$ durch Variation der Vorspannung $U_{v}^{\prime}$ Serienresonanzverhalten $\left(P_{1, \text { min }}\right)$ eingestellt. Die beiden Kapazitäten $C_{\mathrm{j}}(U)$ für $f_{1}$ und $f_{1}^{\prime}$ konnten mit (11) bestimmt werden. Mit den gefundenen Vorspannungen $U_{\mathrm{v}}^{\prime}$ für Serienresonanz bei $f_{1}$ und $f_{1}^{\prime}$ wurde bei den Frequenzen $f_{2}=34 \mathrm{GHz}$ und $f_{2}^{\prime}=34,5 \mathrm{GHz}$ der Reflexionsfaktor nach Betrag und Phase am Anfang des Tapers (Ebene $1-1^{\prime}$ in Bild 3 ) gemessen. Da bei den Frequenzen $f_{2}$ und $f_{2}^{\prime}$ jeweils zwei Reflexionsmessungen mit den bei den Frequenzen $f_{1}$ und $f_{1}^{\prime}$ ermittelten Vorspannungen durchgeführt worden sind, standen zur weiteren Berechnung mit (10) vier Meßwerte zur Verfügung.

Die Auswertung ergab die $C_{\mathrm{p}}$-Werte

$$
\begin{aligned}
& C_{\mathrm{p}, 1}=0,1056 \mathrm{pF}, \\
& C_{\mathrm{p}, 2}=0,1436 \mathrm{pF}, \\
& C_{\mathrm{p}, 3}=0,0834 \mathrm{pF}, \\
& C_{\mathrm{p}, 4}=0,1015 \mathrm{pF} .
\end{aligned}
$$

Das Großsignalverhalten der Diode wurde ebenso mit dem Meßaufbau in Bild 9 untersucht. Für die feste Frequenz $f_{\mathrm{p}}=31 \mathrm{GHz}$ wurde für verschiedene Eingangsleistungen $P_{\text {hin } 1}$ mit der Variation der Vorspannung $(-0,5 \mathrm{~V}$ $\left.\leq U_{\mathrm{v}}^{\prime} \leq 0,5 \mathrm{~V}\right)$ Serienresonanzverhalten $\left(P_{\mathrm{t}, \min }\right)$ eingestellt und die Leistungen $P_{\mathrm{r}, 1}$ und $P_{1,5}$ aus den Meßgrößen $P_{\mathrm{r}}$ und $P_{\mathrm{t}}$ bestimmt. Mit dem Dämpfungsfaktor $D=0,966$ lassen sich dann die in Bild 3 eingezeichneten Leistungen $P_{\text {hin, } 3}, P_{\mathrm{r}, 3}$ und $P_{\mathrm{t}, 3}$ bestimmen.

Der Reflexionsfaktorbetrag

$$
\left|r_{3-3^{\prime}}\right|=\sqrt{\frac{P_{\mathrm{r}, 3}}{P_{\text {hin, } 3}}}
$$

lag für die verschieden großen Eingangsleistungen zwischen $0,904 \leq\left|r_{3-3^{\prime}}\right| \leq 0,909$, und aus

$$
R_{\mathrm{j}}=\frac{Z_{\mathrm{oL}}(0,3)}{2}\left(\frac{1}{\left|\underline{r}_{3-3^{\prime}}\right|}-1\right)
$$

ergibt sich damit ein Bahnwiderstand von $2,17 \Omega \leq R_{\mathrm{j}}$ $\leq 2,3 \Omega$. Mit (16) wurde die Leistung $P_{\mathrm{j}}$ berechnet und damit dann aus (17) der Sperrschichtstrom $I_{\text {j }}$ bestimmt. Gleichzeitig wurde der Strom $I_{\mathrm{j}}$ mit dem in Abschnitt 3.3 beschriebenen Iterationsverfahren ermittelt und mit dem Ergebnis aus (17) verglichen. Die maximale Abweichung zwischen den Sperrschichtströmen $I_{\mathrm{j}}$, die für verschiedene Leistungen $P_{\mathbf{h i n}, 1}$ ermittelt wurden, lag bei $12 \%$.

Mit den ermittelten Diodendaten ließen sich aus (13) und (15) für verschiedene Vorspannungen die Fourierkoeffizienten $S^{(0)},\left|\underline{S}^{(1)}\right|$ und $\left|\underline{S}^{(2)}\right|$ berechnen (Tabelle 1). Die Pumpamplitude wurde so gewählt, daß in etwa immer ein gleicher Richtstrom im nA-Bereich auftrat, so daß der zur Sperrschicht parallel wirkende Leitwert vernachlässigt werden konnte.

Tabelle 1:

\begin{tabular}{lllll}
\hline$U_{\mathrm{v}}[\mathrm{V}]$ & $S^{(0)}\left[\frac{1}{\mathrm{pF}}\right]$ & $\left|\underline{S}^{(1)}\right|\left[\frac{1}{\mathrm{pF}}\right]$ & $\left|\underline{S}^{(2)}\right|\left[\frac{1}{\mathrm{pF}}\right]$ & $q$ \\
\hline$-2,0$ & 8,495 & 0,4371 & 0,01422 & 1,0686 \\
$-1,5$ & 8,057 & 0,4676 & 0,01717 & 1,1432 \\
$-1,0$ & 7,555 & 0,5074 & 0,02158 & 1,2405 \\
$-0,5$ & 6,963 & 0,5631 & 0,02890 & 1,3767 \\
0,0 & 6,224 & 0,6505 & 0,04338 & 1,5903 \\
$+0,5$ & 5,184 & 0,8272 & 0,08574 & 2,0223 \\
\hline
\end{tabular}

Die dynamische Güte $q$ in Tabelle 1 wurde aus (12) für eine Signalfrequenz $f=31 \mathrm{GHz}$ ermittelt $\left(R_{\mathrm{j}}=2,1 \Omega\right)$.

\section{Diskussion der Meßergebnisse}

Die Elemente $C_{\mathrm{j}}(U), L_{\mathrm{p}}$ und $R_{\mathrm{j}}$ der Varaktordiode ließen sich in der Nähe der Serienresonanzfrequenz mit dem Transmissionsmeßverfahren [3] mit guter Genauigkeit für eine Schaltungsdimensionierung bestimmen. Eventuelle Haltereinflüsse werden dabei mit in die Serienkreisdarstellung der Diode einbezogen. Aus der gemessenen $C_{\mathrm{j}}(U)$-Kennlinie erhält man den $C_{\mathrm{j}}(U)$ Kennlinienexponenten $n$ und die Diffusionsspannung $U_{\mathrm{D}}$ der Diode.

Die aus mehreren Reflexionsmessungen erzielten Ergebnisse für die Gehäusekapazität $C_{\mathrm{p}}$ können wegen der Fehlereinflüsse der Reflexionsmessung und der starken Phasenwinkelabhängigkeit der nachfolgenden Berechnungen nur als grobe Abschätzung für eine Schaltungskonzipierung angesehen werden.

Die gute Übereinstimmung zwischen den auf zwei Wegen berechneten Sperrschichtströmen $I_{\mathrm{j}}$ deutet auf eine Stromsteuerung der Schaltung in Bild 9 hin, obwohl sich keine Aussagen über das Oberwellenverhalten des Aufbaus in Bild 9 machen lassen. Die Stromsteuerung war dadurch definiert, da B keine Oberwellen des Sperrschichtstromes auftreten dürfen. Die verwendeten Isolatoren und das koaxiale Filter sind jedoch nicht für Pumpoberwellen ausgelegt. Außerdem sind mit den vorhandenen Meßgeräten quantitative Messungen nur bis maximal $40 \mathrm{GHz}$ möglich. Durch Vorschalten von Bandsperrfiltern bei $f_{p}$ $=31 \mathrm{GHz}$ war es möglich, qualitative Oberwellenleistungsmessungen durchzuführen. Untersuchungen zeigten, daß es für die Praxis genügt, nur die ersten beiden Oberwellen $\mathrm{zu}$ betrachten, da die Fourierkoeffizienten $\underline{S}^{(\eta+1)}(\eta=1,2,3 \ldots)$ für den tatsächlichen Kennlinienverlauf $(n \approx 0,31)$ mit wachsendem $\eta$ sehr schnell vernachlässigbar klein werden. Für diese ersten beiden Oberwellen ist es erforderlich, den kapazitiven Einfluß der Gehäusekapazität durch das Parallelschalten eines kleinen Widerstandes oder einer kleinen Induktivität zu kompensieren, so daß der auf die Pumpfrequenz $f_{\mathrm{p}}$ abgestimmte Serienschwingkreis aus den Elementen $L_{p}, R_{j}$ und $S^{(0)}$ auf einen ohmschen oder jnduktiven Kreis arbeiten kann Die Filtereigenschaften des Serienschwingkreises fuir die Oberwellen sind ausreichend um reg ciner Stromsteguerugg sprechen 
zu können. Dieser Sachverhalt lag vermutlich bei der Großsignalmessung vor, deren mathematische Auswertung mit dem Ansatz einer Stromsteuerung auf verschiedenen Wegen zu etwa gleichen Sperrschichtströmen führte.

Herrn Seidel und Herrn Batzdorfer aus der Institutswerkstatt danke ich für die exakten feinmechanischen Arbeiten.

Diese Arbeit entstand im Rahmen eines von der Deutschen Forschungsgemeinschaft geförderten Forschungsvorhabens.

\section{Literatur:}

[1] Penfield, P.; Rafuse, R.: Varactor Applications. Cambridge Mass., M.I.T. Press, 1960.

[2] Steiner, K.-H.; Pungs, L.: Parametrische Systeme. S. Hirzel Verlag Stuttgart, 1965. Deloach, B. C.: A new microwave measurement technique to characterize diodes and an $800 \mathrm{Gc}$ cutoft frequency varactor at zero volts bias. IEEE Trans. on Microw. Th. and Techn. (1964) 1, S. 15-20.
[4] Schelkunoff, S. A.: Electromagnetic waves. D. Van Nostrand Co., New York, 1943. [5] Bolinder, F.: Fourier transforms in the theory of inhomogeneous transmission lines. Trans. Roy. Inst. Tech., Stockholm, No. 48, 1951.

[6] Collin, R. E.: The Optimum Tapered Transmission Line Matching Section. Proc. IRE (1956) 4, S. 539-548.

[7] Klopfenstein, R. W.: A Transmission Line Taper of Improved Design. Proc. IRE Klopfenstein, R. W.:
(1956) 1, S. 31-35.

[8] Matsumaru, K.: Reflection Coefficient of E-Plane Tapered Waveguides. IRE Trans. on Microw. Th. and Tech. (1958) 4, S. $143-149$.

[9] John, K.: Grundlagen und Bauteile der Mikrowellentechnik. Vorlesungsmitschrift an der TU Berlin, SS 1972.

[10] Rubin, D.: Wide - Bandwidth Millimeter - Wave Gunn Amplifier in Reduced Height Waveguide. IEEE Trans, on Microw. Th. and Techn. (1975) 10, S. 833-834. 11) Marcuvitz, N.: Waveguide Handbook. McGraw-Hill Book Co., Inc., New York, 1951.

[12] Geibler, R.; Chababadi, Z.: Hohlleiter - Filter fir das R-Band $(26,5-40,0 \mathrm{GHz})$ Arch. Elektron. u. Ubertragungstechn. 33 (1979) 1, S. 46-48.

[13] Matthmei, G. L.; Young, L.; Jones, E. M.: Microwave filters, impedance-matching

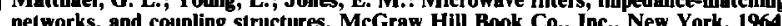
[14] Meinke, H.; Gundlach, F. W.: Teschenbuch der Hochfrequenztechnik. SpringerVerlag, Berlin, 1956.

[15] Whinnery, J. R.; Jamieson, H. W.; Robbins, T. E.: Coaxial-Line Discontinuties. Proc. of the I.R.E. (1944) 11, S. 695-709.

[16] Mohr, W.: Verstimmbarer Hohlleiteroszillator im R-Band. Studienarbeit, U Hannover, Inst. f. HF-Technik, 1979.

Dr.Ing. Rainer Geibler, Hardtweg 7, D-7151 Burgstetten 3

(Eingegangen am 18. 12. 1980)

\section{Tagung}

\section{PRODUCTRONICA 81 in München}

Am Messeplatz München findet vom 10. bis 14. November die PRODUCTRONICA 81 - 4. Internationale Fachmesse für die Fettigung in der Elektronik statt. Begleitet wird dieser Fachtreffpunkt der Elektronik-Fertigungsbranche von einem Fachsitzungsprogramm mit aktuellen Schwerpunktthemen, das den Messeplatz München wieder zur internationalen Informationsbörse für Fachleute der Elektronik-Fertigung in alle Welt werden läßt.

Das Fachsitzungsprogramm, das die Messe vom 9. bis 14. November flankiert, hat folgende thematische Schwerpunkte:

- Aufbau-, Verbindungs- und Montagetechnik des Gerätebaus und

- Meß-, Prüf- und Analysetechniken

Anfragen sind $\mathrm{zu}$ richten an das Kongreßreferat der PRODUCTRONICA 81, Postfach 121009, 8000 München 12.

\section{Neues aus Forschung, Industrie und Wirtschaft}

\section{In den Niederlanden 30 Fernsehprogramme - darunter eines vom} Satelliten

Das Satelliten-Fernsehen ist in den Niederlanden bereits Realität: In Zoetermeer, einer Trabantenstadt von Den Haag, wurden Ende März 30 Fernsehprogramme, darunter auch ein Testprogramm von OTS, dem experimentellen Kommunikations-Satelliten der European Space Agency, in einer Kabelfernsehanlage übertragen. Es handelte sich um die Demonstration der technischen Möglichkeit, schwache Satellitensignale aufzubereiten und in einer Kabelfernsehanlage bis zu 30 Fernsehprogramme zu übertragen. Für den Satellitenempfang hatte Siemens eine 3-mParabolantenne installiert. Das Satelliten-Fernsehprogramm wurde mit 29 weiteren TV-Signalen in einem nach dem MultiKanalkonzept aufgebauten Anlagenabschnitt der Kabelfernsehanlage Zoetermeer eingespeist und über Antennensteckdosen handelsüblichen Fernsehempfängern in herkömmlichen Kanälen zugeführt.

Eine Weltpremiere wie jetzt in Zoetermeer gab es vor zwei Jahren schon in einer anderen niederländischen Gemeinde: Die Bürger von Helmond, einer kleinen Stadt nördlich von Eindhoven, konnten damals über ihre ebenfalls von Siemens eingerichifeid Kabelfernsehanlage 18 Fernsehprogramme und ebenso viele UKW-Hörfunkprogramme empfangen.

\section{Mut zum Bekenntnis für die Technik von morgen}

Ungewißheit bedeute Angst, und es sei eine gemeinsame Aufgabe der Ingenieure in Wissenschaft und Industrie, dieser Ungewi $B$ heit durch zunehmende Aufklärung und durch den Mut zum Bekenntnis für neue technische Entwicklungen entgegenzutreten. Das erklärte Dr.-Ing. Hans Gissel, Mitglied und Leiter der Kommission Forschung und Entwicklung des Vorstands sowie Vorstandsvorsitzender des Unternehmensbereichs Kommunikationstechnik von AEG-Telefunken, aus Anla B der traditionellen Professorenkonferenz des Unternehmens am 5./6. März in Ulm im Hinblick auf die zunehmende Skepsis gegenüber der Technik in der Gesellschaft. „Der Ingenieur in der Industrie muß umweltbewußt arbeiten. Das bedeutet, daß er seine Arbeit in den gesellschaftspolitischen Zusammenhängen erkennen muß.“

Die Schwerpunkte der Professorenkonferenz des Konzerns, an der über 50 Professoren von Universitäten und Technischen Hochschulen sowie Forscher und Entwickler von AEG-Telefunken teilnahmen, waren Vortragsthemen aus der Hochfrequenztechnik und insbesondere der Nachrichtenübertragung. AEG-Telefunken hat im Geschäftsjahr 1980 rd. 1 Milliarde DM für Forschung und Entwicklung aufgewendet. Dr. Gissel betonte die ständig wachsende Bedeutung der Technologie und ihrer raschen Weiterentwicklung für die Strategie des Elektrokonzerns im Zusammenhang mit der Innovation neuer Produkte und ihrer Markterschließung. Dabei sei die enge Zusammenarbeit mit wissenschaftlichen Instituten in Universitäten und Hochschulen wie auch mit Großforschungsanstalten für das Unternehmen von ausschlaggebender Bedeutung.

\section{Private Förderer sollen der Forschung neue Wege ebnen}

Über ihre Meinungen, Einstellungen und Erwartungen zur Wissenschaftsförderung in der Bundesrepublik Deutschland sprach das Düsseldorfer Forschungsinstitut IRES im Auftrag des Stifterverbandes für die Deutsche Wissenschaft mit rund 400 in leitenden Positionen tätigen Wissenschaftlern, Wirtschaftlern, Politikern, Publizisten und Ministerialbeamten. - Die Ergebnisse dieser Untersuchung sind bei einem Pressegespräch des Stifterverbandes am 10. März 1981 in Essen vorgelegt worden.

Sie vermitteln u. a. ein bisher in dieser Deutlichkeit nicht vorhandenes Bild von der für sinnvoll und effektiv gehaltenen „Aufgabenverteilung“" in der Forschungsförderung zwischen Staat, Wirtschaft und privaten Institutionen, das heiBt Stiftungen und gemeinnützigen Vereinen. - Stärken und Schwächen dieser drei Förderungsträger in der Einschätzung der Interviewten werden ebenfalls präzisiert.

Das „Wunschprofil“" für die private Wissenschaftsförderung

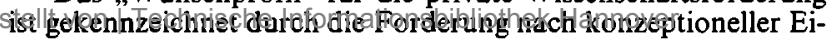
genständigkeit und Risikobereitschaft aufndern Basis rationeller Verwendung der Mittele-Benservineleistungensund dem individu- 